\title{
The sequential changes of the virion polypeptides of CV HB1 virus (Birnaviridae) during serial undiluted passaging
}

\author{
Chu-Fang Lo ${ }^{\mathrm{a}, *}$, Shau-Chi Chi ${ }^{\text {a }}$, Nai-Yueh Tien ${ }^{\text {a }}$, Pei-Jung $\mathrm{Yu}^{\mathrm{a}}$, \\ Chih-Ming Chou ${ }^{a}$, Shiu-Nan Chen ${ }^{a}$, Chung-Hsiung Wang ${ }^{b}$, \\ Guang-Hsiung Kou ${ }^{\text {a }}$ \\ a Department of Zoology, National Taiwan University, Taipei, Taiwan, 107, ROC \\ ' Department of Plant Pathology, National Taiwan University, Taipei, Taiwan, 107, ROC
}

\begin{abstract}
Clam virus (CV HB-1) isolated from hard clam Meretrix lusoria, typed as infectious pancreatic necrosis virus (IPNV) Ab serotype, was employed for the present study. The structural polypeptides of purified virus generated by serial undiluted and diluted passaging were analyzed using sodiumdodecyl-sulfate polyacrylamide gel electrophoresis (SDS-PAGE). The results indicated that in VP2, the virus particles of serial undiluted passaging had viral polypeptides smaller than those of serial diluted passaging. Furthermore, a polypeptide with a molecular mass of $105 \mathrm{kDa}$ was found in the serial undiluted virus preparation. Further analysis by monoclonal antibodies showed that VP105 might be a polyprotein produced by genomic RNA segment $A$. To investigate the sequential changes of viral polypeptides of the virus preparation at high multiplicity infection, the viral polypeptides at each undiluted passage were analyzed by SDS-PAGE followed by immunoblotting. The results indicated that VP105 was present by undiluted passage 7, while the smaller VP2 was present by undiluted passage 10. The amount of smaller VP2 and VP105 increased as the number of passages increased.
\end{abstract}

Keywords: Virion polypeptides, sequential changes; Birnaviridae; Viruses, undiluted passaging; Meretrix lusoria

\section{Introduction}

Infectious pancreatic necrosis virus (IPNV) is an economically important fish pathogen and is the prototype of the Birnaviridae family characterized by a bisegmented, double-

\footnotetext{
* Corresponding author.
} 
stranded RNA genome and an unenveloped, single-layer, icosahedral capsid (Dobos et al., 1979; Brown, 1986). This family also includes infectious bursal disease virus (IBDV) of chicken, Drosophila X virus (DXV) and other aquatic viruses isolated from fish and molluscs (Dobos et al., 1979; Kibenge et al., 1988; Lo et al., 1988; Teninges et al., 1979; Ueno et al., 1984).

There are at least four proteins coded by genomic RNA segment A and B. The large segment A RNA encodes the precursor of major capsid protein (VP2), the internal virion protein (VP3), and the nonstructural protein (NS), and the small segment B RNA encodes the RNA-dependent RNA polymerase (VP1) (MacDonald and Dobos, 1981). Genomic RNA segment A contains a single large open reading frame, capable of encoding $106 \mathrm{~K}$ polyproteins and the genes are arranged in the order of 5'-preVP2-NS-VP3-3' (Duncan and Dobos, 1986; Huang et al., 1986; Nagy et al., 1987). NS protein is an autocatalytic protease and solely responsible for both VP2/NS and NS/VP3 cleavages (Duncan et al., 1987; Manning et al., 1990).

The interference phenomenon exhibited by IPNV was reported by many authors (Nicholson and Dunn, 1974; , Ahne, 1977; MacDonald and Kennedy, 1979; Hedrick and Fryer, 1981, 1982; Hedrick et al., 1986; Lo et al., 1990, 1991). The defective interfering (DI) particles were considered to be responsible for the interference. Successive passage at high multiplicity of infection (MOI) would enhance production of DI particles. Since, to date, it has been impossible to separate DI particles from standard virus (SV) by density gradient centrifugation, we had previously characterized CV HB-1 DI particles by analyzing virion polypeptides and genomic RNA from DI-enriched and SV-enriched virus populations derived from serial undiluted and diluted $\left(10^{-4}\right)$ passaging respectively. A subgenomic RNA segment $A$ and truncated VP2 proteins were detected in DI-enriched virus populations. No differences between DI-enriched and SV-enriched virus preparations were observed in the size of VPl, VP3 and genomic RNA segment B (Lo et al., 1991).

In the present work, we analyzed virion polypeptides at each undiluted passage in order to know the sequential changes of virion polypeptides of the virus propagated at high MOI.

\section{Material and methods}

\section{Cells and viruses}

Clam virus (CV HB-1), an Ab strain of IPNV, isolated from hard clam, Meretrix lusoria, was used in this study (Lo et al., 1988; Lipipun et al., 1988, 1989; Chi et al., 1991). Virus stock was first passaged in TO-2 cells (Chen et al., 1983) by five rounds of limiting dilution. Serial undiluted (MOI $=10^{3} \mathrm{TCID}_{50} /$ cell at initial passage) and $10^{-5}$ diluted $(\mathrm{MOI}=0.01$ $\mathrm{TCID}_{50}$ /cell) passaging of $\mathrm{CV} \mathrm{HB}-1$ virus was carried out with the same procedure as described by Lo et al. (1991) except that the infected cells were incubated at $28^{\circ} \mathrm{C}$. The titer of the released virus (RV) of each passage was estimated in TO- 2 cells at $20^{\circ} \mathrm{C}$ and shown by $\mathrm{TCID}_{50} / 0.1 \mathrm{ml}$.

Analysis of viral polypeptide composition of virus particles from passage 2 of undiluted or $10^{-5}$ diluted passaging

Virus particles with buoyant density of $1.33 \mathrm{~g} / \mathrm{ml}$ in $\mathrm{CsCl}$ were prepared with a procedure described by Lo et al. (1991). Virion polypeptide composition was analyzed with $10 \%$ SDS-PAGE and stained with silver stain. 
Sequential changes of virion polypeptides of virus propagated at high MOI

The cell-associated virus (CAV) from each passage was collected by centrifugation from one $25 \mathrm{~T}$ flask of infected cells, resuspended in $100 \mu \mathrm{l}$ phosphate buffer and divided into ten fractions which were stocked at $-20^{\circ} \mathrm{C}$ before use. The virion polypeptide profiles of the prepared CAV were analyzed by Western immunoblot with the use of polyclonal antibodies produced by hyperimmunization of rabbit with CV HB- 1 virus particles purified from passage 50 of undiluted passaging (Lo et al., 1991).

Analysis of viral polypeptide composition of serial undiluted and $10^{-5}$ diluted virus preparations with monoclonal antibodies (mAbs) against VP2 and VP3

SDS-PAGE minigel system and immunostaining with mAbs after blotting were employed to analyze viral polypeptide profiles of CAV from passage 10 of undiluted and $10^{-5}$ diluted virus preparations. MAbs used in this study included the mixture of four mAbs against VP2 (Eb 1 4) and the mixture of seven mAbs against VP3 (Er 1 7) (Chi et al., 1991, 1993).

\section{Results}

Viral autointerference of $C V$ HB-1 in TO-1 cells under serial passage with high MOI

Fig. 1 shows the comparison of virus in culture fluid from 21 serial passages when undiluted and at $10^{-5}$ dilution. In serial undiluted passaging, the virus yield declined and the virus titer fluctuated from $10^{4.5}$ to $10^{8.4} \mathrm{TCID}_{50} / 0.1 \mathrm{ml}$. The results indicated that serial undiluted passaging of CV HB- 1 could induce autointerference in TO- 2 cells while the virus passaging at $10^{-5}$ dilution showed little interference.

Analysis of viral polypeptide composition of purified virus from passage 21 of undiluted and $10^{-5}$ diluted virus preparations

The structural polypeptides of the purified virus particles of passage 21 from undiluted and diluted virus preparations were analyzed using SDS-PAGE (Fig. 2). The results showed that three viral polypeptides, VP1 (molecular mass $94 \mathrm{kDa}$ ), VP2 (49 kDa) and VP3 (32

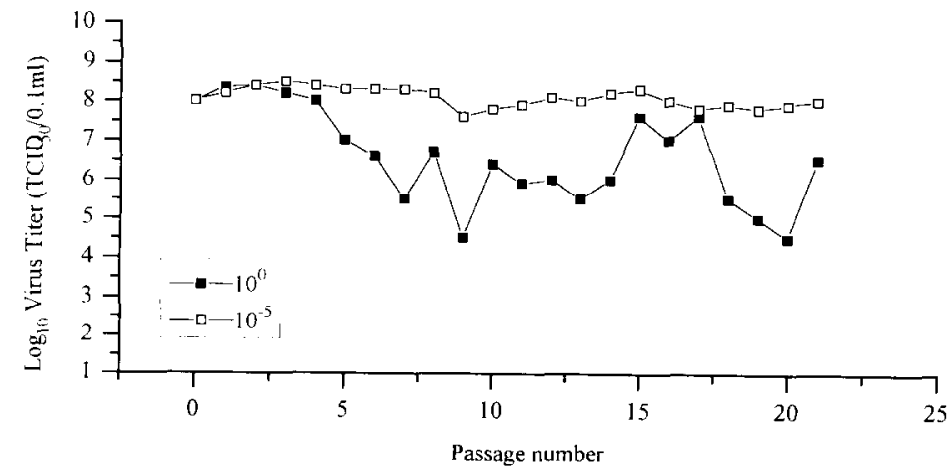

Fig. 1. The comparison of virus in culture fluid from 21 serial passages, undiluted and at $10^{-5}$ dilution. 


\section{3}
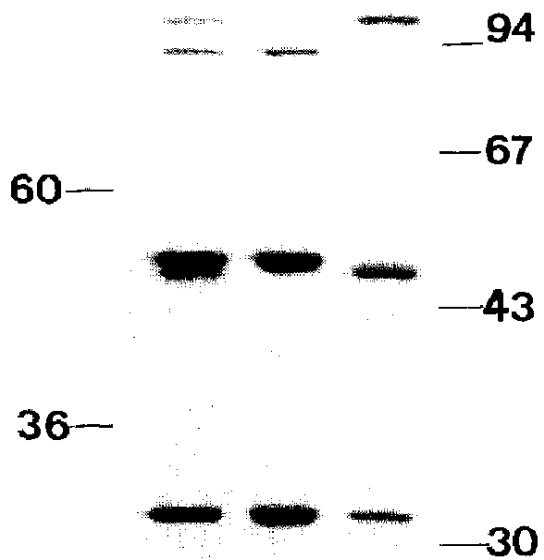

(A)

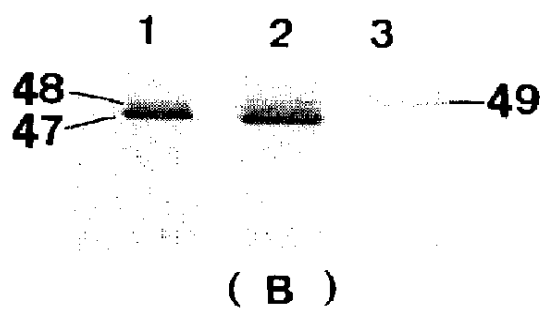

Fig. 2. The structural polypeptide of the purified virus particles of passage 21. (A), VP105, VP1, VP2 and VP3 of viral polypeptides (lane 1 , mixture of diluted and undiluted virus preparations; lane 2, diluted virus preparation; lane 3, undiluted virus preparation); (B), high magnification of VP2 of undiluted virus preparation with a lesser amount of SV loading sample for SDS-PAGE in order to show the VP47, VP48 and VP49 (lanc 1, undiluted virus preparation; lane 2 , mixture of diluted and undiluted virus preparations; lane 3 , diluted virus preparation). The positions of known protein molecular mass markers $\left(\times 10^{3} \mathrm{kDa}\right.$ ) in the same gel are shown on both sides of the photo. 


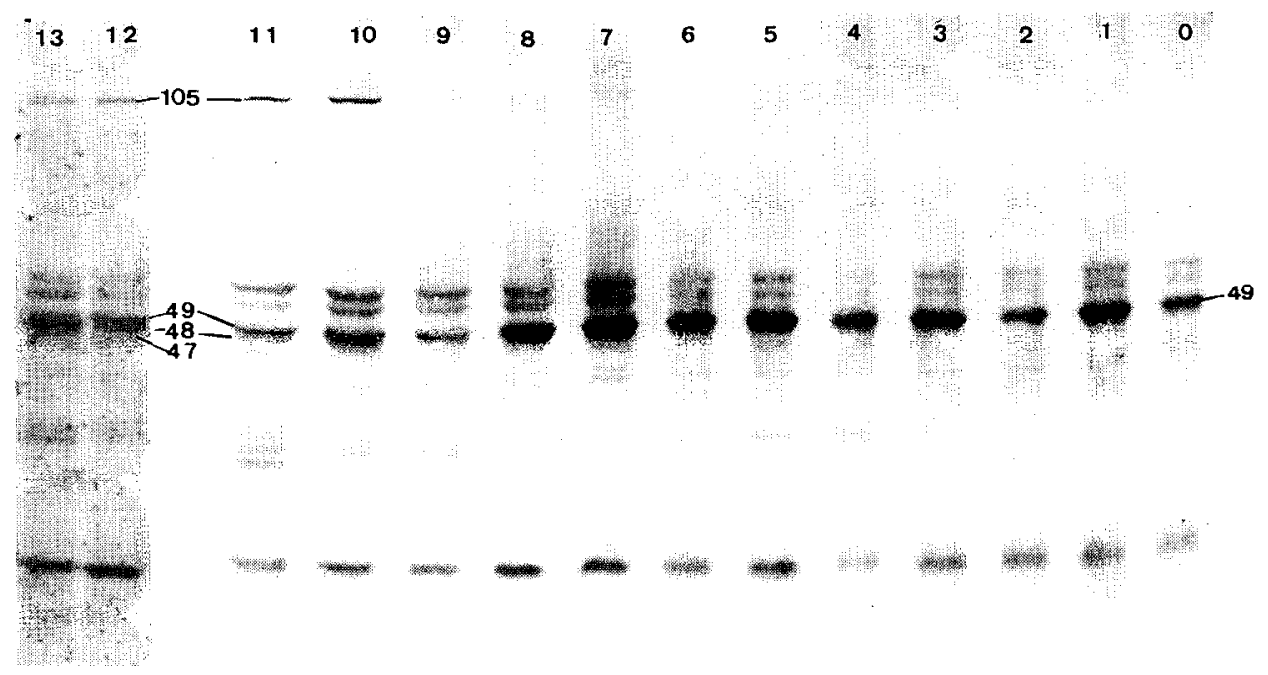

Fig. 3. Western blot analysis of serial undiluted virus preparation showing the sequential change of viral polypeptides during serial undiluted passage. The passage number is shown at the top of the photo and molecular mass $\left(\times 10^{3} \mathrm{kDa}\right)$ of VP47, VP48 and VP105 is also indicated. Passages $0-11$ and passages 12-13 were analyzed in two different gels.

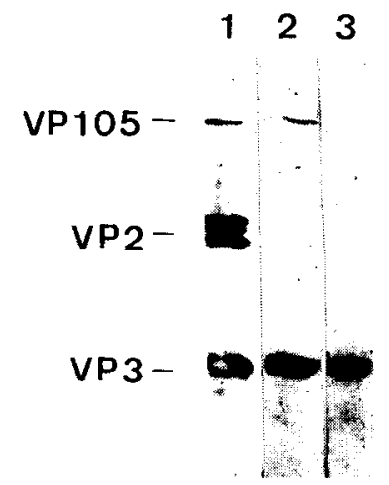

( A )
123

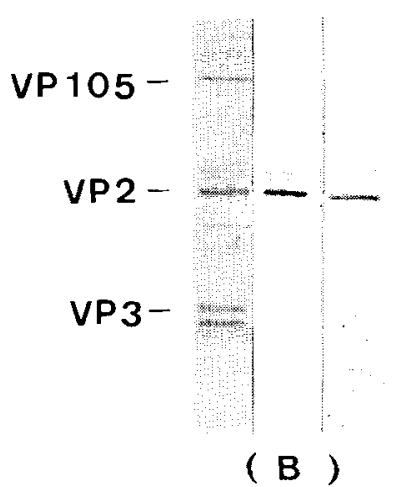

Fig. 4. Analysis of VP105 with mAbs. (A), lane 1, undiluted virus preparation reacted with rabbit polyclonal antibodies; lane 2, undiluted virus preparation reacted with anti-VP3 mAbs; lane 3, diluted virus preparation reacted with anti-VP3 mAbs; (B), lane 1, undiluted virus preparation reacted with rabbit polyclonal antibodies; lane 2, undiluted virus preparation reacted with anti-VP2 $\mathrm{mAbs}$; lane 3 , diluted virus preparation reacted with anti-VP2 mAbs.

$\mathrm{kDa}$ ) were detected in diluted virus preparations, while five viral polypeptides, VP105 (105 $\mathrm{kDa}$ ), VP94, VP48 (48 kDa), VP47 (47 kDa) and VP3 were detected in undiluted virus preparations. VP48 and VP47 were regarded as truncated VP2.

The results indicated that VP2 present in virus particles after serial undiluted passage was a little smaller than VP2 in serial diluted virus preparation. Furthermorc, VP105 was unique in the serial undiluted passaging virus preparation. 


\section{Sequential changes of viral polypeptide profile during serial undiluted passage}

Fig. 3 shows the Western blot analysis of undiluted passaging virus polypeptides. The VP105 was initially detected at passage 7 while the smaller VP2 was initially detected at passage 10. The sequential changes of the viral polypeptides in the subsequent passage were readily observed. The amount of those smaller VP2 and VP105 increased as the number of passages increased.

\section{Analysis of VPIO5 with mAbs against VP2 and VP3}

Since VP105 was unique in serial undiluted virus preparation, further analysis with mAbs against VP2 and VP3 was carried out in order to know the nature of VP105 (Fig. 4). The results indicated that VP105 could react with anti-VP3 mAbs, but not with anti-VP2 mAbs, and that VP105 contained part of, if not all, VP3.

\section{Discussion}

The virus for the present study was isolated from the gill of hard clam ( $M$. lusoria) and was defined as Ab serotype based on its biochemical properties, reciprocal neutralization test and mAb reaction patterns (Lo et al., 1988; , Chi et al., 1991). However, CV HB-1 could be distinguished from Ab type strain by mAb E3 (Caswell-Reno et al., 1989; Lipipun et al., 1989). In our previous studies, we have found that the autointerference of CV HB-1 always occurred in TO-2 cells infected with the virus at high MOI and the DI particles were considered to be responsible for the interference (Lo et al., 1990). By comparing the polypeptides and RNA in virus particles purified from $10^{4}$ diluted and undiluted virus preparations at passages 40 and 50, it was revealed that (1) the virus particles of undiluted virus preparation had VP2 polypeptides smaller than those of standard virus, and (2) subgenomic segment A RNA was found in virus particles of undiluted virus preparation. This evidence suggested that DI particles of CV HB-1 was a deletion mutant of standard virus (Lo et al., 1991). Lowered affinity of VP2 to mAbs in serial undiluted passage of CV HB-1 was also observed, which indicated some changes did occur on VP2 during the serial undiluted subcultures (Chi et al., 1993).

In the present study, we report for the first time the sequential changes of viral polypeptides during serial subcultures at high MOI. Furthermore, we have also partially characterized the protein, VP105, which was unique in undiluted virus preparations. VP105 could be recognized by anti-VP3 $\mathrm{mAbs}$ but failed to be recognized by anti-VP2 $\mathrm{mAbs}$ in the present study. However, more antibodies against VP2 and NS should be prepared in order to further investigate the nature of VP105.

The genome A-segments of IPNV and IBDV each contained a single open reading frame of approximately 2900 bases (Duncan and Dobos, 1986; Hudson et al., 1986). It was reported that a polyprotein containing NH3-preVP2-NS-VP3-COOH was synthesized first by transcription of the A-segment and that the proteolytic activity of NS was involved in the processing of this polyprotein (Huang et al., 1986; Hudson et al., 1986; Manning et al., 1990). The polyprotein had been found in cell-free translation of A-segment transcripts of IPNV (Duncan et al., 1987) and the bacterial expression of the A-segment of IBDV (Azad et al., 1987; Jagadish et al., 1988), but had never been observed in the infected cells, 
possibly due to the fast processing of the polyprotein to preVP2, NS, and VP3. From the size of VP105 found in the present study, it is highly possible that VP105 is a polyprotein translated from A-segment transcript. Mutations may occur in VP105 which may prohibit the protein from being processed to preVP2, NS and VP3, and, thus, retained in the infected cells.

\section{Acknowledgements}

This research was supported by the National Science Council (NSC 810418-b-002-09), ROC.

\section{References}

Ahne, W., 1977. Persistent infection in CHSE-214 cells with IPN virus isolated from pike (Esox lucius). Bull. Off. Int. Epizoot., 87: 415-417.

Azad, A.A., Jagadish, M.N., Brown, M.A. and Hudson, P.J., 1987. Deletion mapping and expression in Escherichia coli of the large genomic segment of a birnavirus. Virology, 161: 145-152.

Brown, F., 1986. The classification and nomenclature of viruses: summary of results of meeting of International Committee on Taxonomy of Viruses in Sendai. September, 1984. Intervirology, 25: 140-143.

Caswell-Reno, P., Lipipun, V., Reno, P.W. and Nicholson, B.L., 1989. Use of a group-reactive and other monoclonal antibodies in an enzyme immunodot assay for identification and presumptive serotyping of aquatic birnaviruses. J. Clin. Microbiol., 27: 1924-1929.

Chen, S.N., Chi, S.C., Ueno, Y. and Kou, G.H., 1983. A cell line derived from tilapia ovary. Fish Pathol., 18: 1318.

Chi, S.C., Chen, S.N. and Kou, G.H., 1991. Establishment, characterization and application of monoclonal antibodies against Eel Virus European (EVE). Fish Pathol., 26: 1-7.

Chi, S.C., Lo, C.F., Chen, S.N. and Kou, G.H., 1993. Lowered affinity of structural protein VP2 to monoclonal antibodies in serial undiluted passage of infectious pancreatic necrosis virus (IPNV). Fish. Pathol., 28(1): $51-52$.

Dobos, P., Hill, R.J., Hallet, R., Kells, D.T.C., Becht, II. and Teninges, D., 1979. Biophysical and biochemical characterization of five animal viruses with bisegmented RNA genomes. J. Virol., 32: 593-605.

Duncan, R. and Dobos, P., 1986. The nucleotide sequence of infectious pancreatic necrosis virus (IPNV) dsRNA segment A reveals one large ORF encoding a precursor polyprotein. Nucleic Acids Res., 14: 5934

Duncan, R., Nagy, E., Krell, P.J. and Dobos, P., 1987. Synthesis of the infectious pancreatic necrosis virus polyprotein, detection of a virus-encoded protease, and fine structure mapping of genome segment A coding regions. J. Virol., 61: 3655-3664.

Hedrick, R.P. and Fryer, J.L., 1981. Persistent infection of three salmonid cell lines with infectious pancreatic necrosis virus (IPNV). Fish Pathol, 15: 163-172.

Hedrick, R.P. and Fryer, J.L., 1982. Persistent infections of salmonid cell lines with infectious pancreatic necrosis virus (IPNV): a model for the carrier state in trout. Fish Pathol, 16: 163-172.

Hedrick, R.P., Eaton, W.D., Fryer, J.L., Groberg, W.G. Jr. and Boonyaratapalin, S., 1986. Characteristics of a birnavirus isolated from cultured sand goby Oxyeleotris marmoratus. Dis. Aquat. Org., 1: 219-225.

Huang, M.T.F., Manning, D.S., Warner, M., Stephens, E.B. and Leong, J.C., 1986. A physical map of the viral genome for infectious pancreatic necrosis virus $\mathrm{Sp}$ : analysis of cell-free translation products derived from viral cDNA clones. J. Virol, 60: 1002-1011.

Hudson, P.I., Mcken, N.M., Power, B.E. and Azad, A.A., 1986. Genomic structure of the large RNA segment of infectious bursal disease virus. Nucleic Acids Res., 14: 5001-5012.

Jagadish, M.N., Staton, V.J., Hudson, P.J. and Azad, A.A., 1988. Birnavirus precursor polyprotein is processed in Escherichia coli by its own virus-encoded polypeptide. J. Virol., 62: 1084-1087. 
Kibenge, F.S.B., Russell, R.G. and Dhillon, A.S., 1988. Biochemistry and immunology of infectious bursal disease virus. J. Gen. Virol., 69: 1757-1775.

Lipipun, V., Caswell-Reno, P. and Nicholson, B.L., 1988. Enzyme immunoassay utilizing monoclonal antibodies for identification of European eel virus (EEV), an aquatic birnavirus. In: C. Yentsch, F. Magues and Ph. Horan (Editors), Immunological Approaches to Coastal, Estaurine and Oceanographic Questions - Lecture Notes on Coastal and Estuarine Studies \#25. Spring-Verlag, pp. 312-317.

Lipipun, V., Caswell-Reno, P., Hsu, Y.L., Wu, J.L., Tung, M.C., Reno, P.W., Wattanavaijarn, W. and Nicholson, B.L., 1989. Antigenic analysis of Asian aquatic birnavirus isolates using monoclonal antibodies. Fish Pathol., 24: $155-160$.

Lo, C.F., Hong. Y.W., Hung, S.Y. and Wang, C.H., 1988. The characteristics of the virus isolated from the gill of clam, Meretrix lusoria. Fish Pathol., 23: 147-154.

Lo, C.F., Lin, M.S., Liu, S.M., Wang, C.H. and Kou, G.H., 1990. Viral interference in TO-2 cells infected with IPN virus isolated from clam, Meretrix lusoria. Fish Pathol., 25: 133-140.

Lo, C.F., Wang, C.H., Lin, M.S. and Kou, G.H., 1991. Viral polypeptides and RNA of defective interfering particles of IPN virus generated by serial undiluted passaging in TO-2 cells. Fish Pathol., 26: 119-125.

MacDonald, R.D. and Dobos, P., 1981. Identification of the proteins encoded by each genome segment of infectious pancreatic necrosis virus. Virology, 114:414-422.

MacDonald, R.D. and Kennedy, J.C., 1979. Infectious pancreatic necrosis virus persistently infects chinook salmon embryo cells independent of interferon. Virology, 95: 260-264.

Manning, D.S., Malson, C.L. and Leong, J.C., 1990. Cell-free translational analysis of the processing of infectious pancreatic necrosis virus polyprotein. Virology, 179: 9-15.

Nagy, E., Duncan, R., Krell, P. and Dobus, P., 1987. Mapping of the large RNA genome segment of infectious pancreatic necrosis virus by hybrid arrested translation. Virology, 158: 211-217.

Nicholson, B.L. and Dunn, J., 1974. Homologous viral interference in trout and Atlantic salmon cell cultures infected with infectious pancreatic necrosis virus. J. Virol., 14: 180-182.

Teninges, D., Ottanessian, A., Richard-Molard, C. and Contamine, D., 1979. Isolation and biological properties of drosophila $X$ vrius. J. Gen. Virol., 42: 241-254.

Ueno, Y., Chen, S.N., Kou, G.H., Hedrick, B.P. and Fryer, J.L., 1984. Characterization of virus isolated from Japanese eel (Anguilla japonica) with nephroblastoma. Bull. Inst. Zool, Acad. Sin., 23: 47-55. 